\title{
The drugs and application methods in dental sedation
}

\author{
Fatih Oznurhan* and Cansu Derdiyok \\ Sivas Cumhuriyet University, Faculty of Dentistry, Department of Pediatric Dentistry, Turkey
}

\begin{abstract}
Sedation and general anaesthesia is indicated when routine dental treatments cannot be performed due to fear and anxiety in dentistry. The agents selected for sedation are administered by different routes such as inhalation, oral, intramuscular (IM), intravenous (IV), intranasal (IN), sublingual (SL) and rectal. Each of these routes of administration has the potential to produce sedation when the appropriate agent is selected. In this article, it is aimed to give information about sedation administration routes and drugs used in sedation.
\end{abstract}

\section{Introduction}

Ensuring anxiety and fear control of patients during dentistry is one of the most difficult problems [1]. Most of the pediatric patients do not adapt to dental treatment because their comprehension skills are not fully developed, and they do not understand enough of a complex event such as dental treatment [2]. Communication and behavior guidance techniques do not work, especially in patients who are under-age and who cannot perceive the necessity of treatment [3]. Pharmacological methods may be needed in pediatric patients to perform dental treatments when basic behavior guidance methods are not sufficient and to ensure patient compliance. Conscious sedation (low / moderate sedation) is the technique where drugs are used to cooperate with anxious and incompatible children and the patient is conscious. Contrary to this situation in which the patient is awake and conscious, deep sedation and general anesthesia are characterized by partial or complete loss of protective reflexes of the patient [4].

It is predicted that the prevalence of dental fear throughout the society will decrease over the years. With fewer children requiring extensive dental treatment, the learned fear of dentists during childhood will be reduced. Nevertheless, tooth decay remains a common problem in childhood and is particularly prominent in low-income populations. These facts show that the need and demand for sedation will gradually increase in order to alleviate anxiety and fear because dental practices are still perceived as a source of pain [5].

\section{Sedation application ways}

Agents selected for sedation; inhalation, oral, intramuscular (IM), intravenous (IV), intranasal (IN), sublingual (SL) and rectal. Each of these routes of administration has the potential to produce sedation when the appropriate agent is selected [6].

\section{Inhalation}

Inhalation is the route of sedation where the gas or volatile sedative agent reaches the alveoli in the lungs and shows its primary effect by absorption via blood-gas [7]. The agent that reaches the alveoli reaches the tissues through the arteries, arterioles and capillaries by mixing into the blood from the alveoli and shows its effect in the central nervous system [8]. It is the safest technique that used most frequently in children [9]. Many agents such as nitrous oxide, isoflurane, sevoflurane and desflurane are used in inhalation sedation. Inhalation agents other than nitrous oxide are generally used in deep sedation and general anesthesia [10]. The most commonly used sedation in dentistry is nitrous oxide inhalation sedation, which can be used alone or in combination with other sedative agents [11].

\section{Oral}

The oral route is an easy and economical way to administer and is the oldest used drug administration route. Although other routes of drug administration are more effective and reliable in achieving the desired clinical effect, the oral route still has a valuable place in the face of pain and anxiety in dentistry [12]. The advantages of oral sedation are ease of administration, relatively safe, easy acceptance by the patient, low cost, low side effects, and no need for needles, syringes or equipment. However, long onset of action, irregular and incomplete absorption from the gastrointestinal tract, inability to titrate, sedation levels cannot be easily changed [13]. Although oral sedation seems to be easier compared to other sedation techniques, this is limited only by its administration, and adjusting the type and dosage of the drug requires special care [14].

\section{Intramuscular}

Intramuscular administration is a parenteral technique in which the drug enters the cardiovascular system directly without passing through the gastrointestinal tract. In parenteral techniques, parenteral techniques are advantageous over enteral techniques (oral, rectal) since

*Correspondence to: Fatih Oznurhan, Sivas Cumhuriyet University Faculty of Dentistry Department of Pediatric Dentistry, Kampus-Merkez Sivas, Turkey, Tel: 0090346219 1010- 3103; Fax: 009034621912 37; E-mail: fatihozn@ hotmail.com

Key words: dentistry, sedation, inhalation, nitrose oxide/ oxygen

Received: January 03, 2020; Accepted: January 13, 2020; Published: January 20,2020 
the drug does not have to enter the enterohepatic circulation before entering the systemic circulation. Intramuscular administration is a less preferred method of administration than other parenteral applications of inhalation and intravenous methods in dentistry.

Advantages of intramuscular administration; the rapid onset of the drug (about $15 \mathrm{~min}$ ), the faster the highest clinical effect (about $30 \mathrm{~min}$ ) and the more reliable absorption of the drug than enteral administration. The disadvantage of intramuscular administration is that there is a latent time of 10 minutes that does not allow titration, that the effect of the drug cannot be reversed rapidly when various complications develop, that patients are not willing to inject, long duration of action (about 2-4 hours or more) and injuries that may occur during injection. Intramuscular method is not recommended for conscious sedation in pediatric patients [15].

\section{Intravenous}

Intravenous administration is the fastest mode of action of the drug, and hand-heart-brain circulation is completed in approximately 20-25 seconds. The most important advantage of intravenous administration is that the drug can be titrated. This reduces the risk of respiratory depression. Another advantage of titration is that it allows the desired sedation level to be achieved according to the patient and the type of treatment planned, and the risk of insufficient or overdose medication is low [16]. The duration of reversal of the effect of intravenous drug administration; it is short compared with oral, rectal, intranasal and intramuscular methods, but longer compared with nitrous oxide / oxygen inhalation. For continuous intravenous infusion recommended for sedation administration, vascular access is required throughout treatment. The major advantage of open vascular access is that it allows for the rapid administration of any medication that may be required in the event of an emergency [17]. Side effects such as nausea and vomiting in intravenous administration are rare. Salivary secretion can be controlled by intravenous anticholinergic agent. Although nausea reflex decreases with intravenous administration, the first choice in patients with nausea reflex is nitrous oxide / oxygen inhalation [18]. Vascular access is a great advantage for drug administration, whereas pediatric patients do not comply, and small vessels make it difficult to administer. Complications such as hematoma, phlebitis and intraarterial injection of the drug can be seen in the vascular site [19]. Scottish Intercollegiate Guidelines Network (SIGN), Since there is not enough scientific evidence to support the routine use of intravenous sedation for dental treatment in children under the age of 16 , it does not recommend conscious sedation administered intravenously in children, except in special cases, and states that intravenous administration should be performed in a hospital setting when necessary [20].

\section{Intranasal}

Intranasal administration, which has recently begun to be used with increasing interest in non-invasive applications, is used as an alternative to sedation by oral or injection, especially in paediatric patients [21]. Intranasal administration is a painless, inexpensive and easy to apply method [22]. The duration of onset of action in intranasal administration is close to intravenous administration [21].

\section{Sublingual}

The advantages of this method are that the permeability of the mucosa in the sublingual region is very high and that the drug passes directly into the systemic circulation with virtually no enterohepatic circulation. In this way, the first-pass effect of the liver where biotransformation of a part of the drug is performed is prevented [23]. The necessity of patient compliance for application limits the use of the method in younger and non-compliant patients [21].

\section{Rectal}

The availability of more reliable drug administration routes reduces the frequency of rectal administration. Although it is reported that it can be used in very young children or patients with disabilities where the oral route cannot be administered, rectal sedation is generally not preferred because of the discomfort of the individual and patient, changing absorption of certain drugs in the large intestine, regional irritations, and the disadvantage that the drug cannot be easily reversed [24].

\section{Drugs used in sedation}

\section{Hydroxyzine}

The Hydroxyzine is an antihistamine that has features of reducing sedative, antiemetic and salivary secretion [4]. It has no effect on respiratory and circulatory system in normal doses. After oral administration, it is rapidly absorbed from the gastrointestinal tract and has a clinical effect in 15-30 minutes. The highest clinical effect occurs in 2 hours and lasts approximately 3-4 hours. Because of these properties, it has been used in sedation procedure in paediatric patients for many years [25]. Oral administration may be preferred. It is not preferred especially in paediatric patients because of intramuscular administration difficulties. Intravenous and subcutaneous injections are not recommended due to the risk of tissue necrosis and haemolysis. There are two forms of hydroxyzine hydrochloride and hydroxyzine pamoate [12]. Oral hydroxyzine is generally used together with other agents such as chloral hydrate, meperidine, midazolam, nitrous oxide to form an effective sedation [26].

\section{Promethazine}

Promethazine is a phenothiazine with sedative and antihistaminic properties. Absorption is good when taken orally. It can be administered intramuscularly and very rarely by intravenous route. The onset of action is 15-60 minutes, peaks in 1-2 hours and the duration of action is 4-6 hours. It should be used with caution in children with a history of asthma, sleep apnoea and family history of sudden infant death syndrome. Promethazine should not be used in seizure-prone patients because it reduces the seizure threshold. All phenothiazines cause some central nervous system depression. The effect of this group of drugs is different from barbiturates, benzodiazepines and other sedative-hypnotic drugs. The fact that phenothiazines do not cause unconsciousness, respiratory and circulatory system depression and addiction at high doses are two important differences of this group of drugs [12]. Phenothiazines are often used in combination with agents such as meperidine, chloral hydrate, ketamine [27]. The most commonly reported side reactions are dry mouth, blurred vision, mild hypotension, darkening of bronchial secretions. The most important side reactions are extrapyramidal reactions [12].

\section{Diazepam}

Diazepam is an oil-soluble and water-insoluble long-acting benzodiazepine. When taken orally, it is rapidly absorbed from the gastrointestinal tract and reaches the highest plasma level in 2 hours, after intravenous administration it is 1-2 minutes. When the drug is taken orally, it is recommended to take the drug one hour before the procedure, since $90 \%$ of the highest clinical effect occurs within 1 hour. 
Diazepam is very effective in relieving mild and moderate anxiety. The rapid onset of action makes it suitable for dental treatment. The effect on cardiovascular system in healthy individuals is minimal. As with all existing sedative-hypnotic drugs, there is a danger of respiratory depression [4]. Diazepam causes anterograde amnesia when administered intravenously and this period varies between individuals, but it is about 10 minutes [28]. Amnestic effect is usually not seen when the drug is administered orally and intramuscularly. In the amnestic phase, traumatic procedures such as local anaesthesia may be completed [12]. When diazepam is used for low or moderate sedation, the expected side reactions are only ataxia and prolonged central nervous system effects. Optimal sedation cannot be achieved when intravenous diazepam is administered alone for dental treatment in a paediatric patient. While the adult patient is more calm and conformist, paradoxical reactions such as fighting, and agitation can be seen in paediatric patients. Oral administration is not recommended before 6 years of age [4].

\section{Midazolam}

Imidazobenzodiazepine is a benzodiazepine group with strong sedative, amnesic and anxiolytic properties. It has a faster onset time and a shorter duration of action than other benzodiazepines. It can be administered by oral, intranasal, intravenous, intramuscular and rectal routes [30]. It is the ideal sedative agent present in paediatric patients because of its features such as oral and intranasal administration, anxiolytic and anterograde amnestic effects and short duration of action [29]. When administered orally, the intravenous preparation is difficult to use due to the bitter taste. It can be used with various fruit juices, sodium citrate and sweeteners such as Ora-sweet, acetaminophen elixir or kool-aid to mask the bitter taste. It should be noted that the $\mathrm{pH}$ and effectiveness of midazolam may vary when used with these sweeteners. Therefore, the child should be allowed to swallow the drug at once. In case of sip, the child may refuse the drug $5 \mathrm{mg} / 5 \mathrm{~mL}, 15 \mathrm{mg} / 3$ $\mathrm{mL}, 50 \mathrm{mg} / 10 \mathrm{~mL}$ ampoules and Dormicum ${ }^{\star}$ (Roche) $7.5 \mathrm{mg}$ tablets and Versed ${ }^{\circ}$ (Roche) $2 \mathrm{mg} / \mathrm{mL}$ in the world under the trade name Dormicum $^{\oplus}$ (Roche) syrup-118 $\mathrm{mL}$ in the oral form is in the bottle [31]. Midazolam is highly soluble in water and does not irritate the veins. It is a powerful sedative amnesic when used intravenously or in combination with a narcotic. Midazolam, like all benzodiazepines, does not alleviate pain perception while changing the response to pain. It has the advantage of providing forward and backward amnesia. Paradoxical reactions may occur in paediatric patients as a result of midazolam use. These reactions are hallucinations, disorientation, continuous crying, continuous movement and in some cases the desired level of sedation cannot be achieved, even agitation (delirium) is the development. Therefore, the midazolam dose should be adjusted well. Higher doses of midazolam may cause hypotension, especially in hypovolemic children. At low doses, the patient's respiratory reflexes are maintained while anxiety alleviates and the patient calms down.

Although the most effective route is intravenous, oral premedication is preferred because children respond to needle exaggerated psychological intravenous reactions. Diazepam is preferred when midazolam is administered intravenously. It is 3-4 times more potent than diazepam and switches from the initial effect to the peak effect within 2-3 minutes after intravenous administration. The efficacy of midazolam in paediatric patients has been proven in many studies [31]. The most serious side reactions of midazolam in paediatric patients are hypoventilation, apnoea, laryngospasm, hypotension, cardiac arrest and anaphylaxis. Nausea, vomiting, dizziness, headache, diplopia and paradoxical reactions are the more common minor side reactions [30].
Effect of midazolam on systems:

- Cardiovascular System: Cardiovascular depression is minimal in induction doses. Arterial pressure, cardiac output and peripheral vascular resistance show little decrease. Sometimes it can increase the number of heart beats. Midazolam is considered to lower blood pressure and peripheral resistance more than diazepam.

- Respiratory system: It inhibits the respiratory reaction to carbon dioxide. This depression is important in intravenous administration or when combined with other drugs that cause respiratory depression. Although apnoea is less than induction of barbiturate, it can also be observed in intravenous administration.

- Cerebral: Cerebral reduces oxygen consumption and blood flow. It is very effective in epileptic seizures. Oral sedative doses constitute anterograde amnesia. Their muscle relaxation is at the spinal level. Induction doses may cause loss of consciousness. No direct analgesic effects.

- Drug interactions: Erythromycin inhibits midazolam metabolism, prolongs its effect and intensifies. This antibiotic inhibits the destruction of oral midazolam in the liver, leading to an increase in plasma levels, thus increasing the severity and duration of the sedative effect. Ethanol, barbiturate and other agents that causes central depression strengthen the effect of midazolam [32].

\section{Meperidine}

Meperidine, an opioid agonist, has been used mainly for intravenous sedation for many years. Opioids are preferred because of their very strong analgesic properties. Although it affects many systems in the body, its therapeutic properties are due to its effects on the central nervous system. Opioids cause analgesia, drowsiness, changes in mood and blur the mind, but do not cause loss of consciousness [33]. Meperidine is the only opioid that causes tachycardia and reduces secretions. May cause orthostatic hypotension. It is not recommended to be administered in individuals with severe asthma due to respiratory depression. Other side reactions include dysphoria, nausea and vomiting. At therapeutic doses, it has almost no adverse effects on the cardiovascular system [32]. It is used together with other central nervous system depressants because it requires high doses for sedation alone. In paediatric dentistry, the use of meperidine in combination with promethazine, which is often antihistamine, is preferred. Tolerance and dependence may develop after repeated applications. Although its potential to cause dependence in the use of sedation in dentistry is quite low, this feature is a limiting factor in the choice of meperidine [15].

\section{Fentanyl}

Fentanyl is a short-acting opioid agonist with a fast onset time. Its clinical effect is similar to meperidine and morphine. However, $1 \mathrm{mg}$ fentanyl provides analgesia equivalent to $100 \mathrm{mg}$ morphine and $750 \mathrm{mg}$ meperidine. Fentanyl has replaced meperidine for moderate intravenous sedation in dentistry. In intravenous administration, the analgesia and sedation effect start in less than 1 minute and the clinical effect lasts for an average of 30-60 minutes. In intramuscular administration, the effect starts in 5-15 minutes, the maximum effect develops in 30 minutes and the duration of action is about 1-2 hours. Fentanyl is a useful drug for the relief of pain and anxiety by intravenous or intramuscular route for short-term dental and surgical procedures [34]. Fentanyl, like other opioid agonists, may cause myosis, bradycardia bronchoconstriction and euphoria, rarely nausea and vomiting Fentanyl-induced respiratory depression lasts longer than its analgesic effect [15]. 


\section{Chloral hydrate}

Chloral hydrate is one of the hypnotic group drugs synthesized in 1832. With the introduction of effective drugs such as midazolam in pediatric dentistry, the use of chloral hydrate has decreased. Oral capsule, elixir form and rectal suppository is available. It is an effective drug in patients with low and moderate anxiety [15]. Drowsiness and drowsiness usually pass in $30-45$ minutes and the total effect time is 2-5 hours [35]. Chloral hydrate can cause irritation to the skin and mucosa. It also irritates the gastrointestinal tract in the vast majority of patients. Gastric discomfort can be reduced by diluting the drug or by drinking a glass of water or milk after the drug. It does not have analgesic properties. The effect of chloral hydrate on blood pressure and respiration at therapeutic doses is similar to that in normal sleep. Disturbing side reactions of chloral hydrate include bad taste, stomach upset, nausea and vomiting. Other central nervous system effects are dizziness, ataxia, and nightmares [15].

When the desired effect cannot be achieved with the dose taken, it can be used in combination with nitrous oxide / oxygen inhalation to increase efficacy. Since titration of inhalation sedation is possible, the level of sedation sought can be achieved in this way. If the oral dose is not sufficient, the dose should not be increased, or a different agent should be given orally. In another session, the procedure should be performed by re-calculating the dose according to the patient's response. In order to obtain maximum benefit from chloral hydrate, the appointment period should not be longer than 1 hour [35]. Although chloral hydrate is a safe drug with minimal effect on respiration, it can have serious effects that may cause permanent neurological damage and death when given in high doses, not given in hospital environment, administered by untrained people and when the patient is not observed enough and discharged quickly [36].

\section{Ketamine}

Ketamine is an aesthetic agent which has been preferred for many years in anaesthesia practice and has analgesic, hypnotic and amnesic effects. The condition that ketamine creates by creating electrophysiological dissociation between cortical and limbic system is called 'dissociative anaesthesia. This prevents the patient from perceiving visual, auditory and painful stimuli, and presents a clinical picture in which the patient stands awake, his eyes are open and can move involuntarily, but appear to be unaware of the environment. Since ketamine is water and oil soluble, it can be administered by intravenous, intramuscular, oral, rectal, intranasal routes. While bioavailability is $90 \%$ after intravenous administration, it decreases to $16 \%$ in oral administration. The highest plasma concentration is reached approximately 1 minute after intravenous administration, 5 minutes intramuscular administration and 30 minutes after oral administration. Ketamine has a sympathomimetic effect on the cardiovascular system, causing an increase in blood pressure, heart rate and heart rate. After the application of ketamine, spontaneous respiration continues, and muscle tone of the upper respiratory tract increases. Pharyngeal and laryngeal airway reflexes are usually preserved. Ketamine acts as a bronchodilator. Ketamine stimulates saliva and tracheobronchial secretions that may cause laryngospasm. The use of oropharyngeal tampon is recommended when ketamine is used during dental treatments [37].

Wathen et al. [38] compared only ketamine and ketamine as well as midazolam patients. Vomiting was higher in the ketamine-treated group than in the ketamine and midazolam-treated group. At the same time, vomiting was observed more frequently in children who received ketamine under 10 years of age compared to children who received ketamine over 10 years of age. Ketamine can cause skeletal muscle hypertonicity and rigidity. This effect can be reduced by administration of benzodiazepine. Random movements that are not associated with painful stimuli may be seen in the patient. These movements should not be confused with insufficiency of sedation level [37]. Ketamine should be administered only by an experienced anesthesiologist in a hospital setting [35].

\section{Propofol}

Propofol is the most commonly used intravenous anaesthetic agent for induction of anaesthesia, which is not chemically related to other intravenous anaesthetics. It has no analgesic effect and can be used for sedation in doses lower than the dose required for general anaesthesia. It has a narrow security range when used for sedation [39]. It is preferred in sedation applications due to its short duration of action and rapid recovery, as well as its low side reaction [33]. It is a strong respiratory depressant. Since it does not cause histamine release, it can be used safely in patients with asthma. Propofol is also known for its amnestic and antiemetic properties. During injection, it may cause pain. To prevent injection pain, it is recommended that the drug be injected slowly into a large vein and administered prior to intravenous lidocaine [40]. Propofol should not be used in children with egg allergy due to its formulation containing egg lecithin [34]. Since the safety and efficacy of propofol use in children has not been established, its use is not recommended [33]. In case of application, it is recommended to be applied in a hospital environment by an experienced anaesthesiologist [39].

\section{Nitrous oxide / oxygen}

Nitrous oxide is used to provide general anaesthesia in the 1800 s, analgesia in the 1940-50s and conscious sedation today [41]. Hulland et al. [42], a 10-year retrospective study of 819 paediatric patients, nitrous oxide / oxygen gas was the most effective, safe and preferred sedation agent. In the study of Işı et al. [43] reported that nitrous oxide / oxygen gas used in combination with midazolam increases the effectiveness of sedation and is more preferred to paediatric patients. Nitrous oxide is a non-irritating, pleasantly smelling, colourless gas obtained by heating the ammonium nitrate crystals to $240^{\circ} \mathrm{C}$. It is the only inorganic gas used to create anaesthesia in humans. When the nitrous oxide gas is inhaled, it is rapidly absorbed from the alveoli and transported by physical dissolution without binding to any element in the blood when it enters the circulation [43]. Because of its low solubility in blood, the onset of action and recovery time are very fast. Although nitrous oxide is classified among inhalation anaesthetics, it is the weakest and provides limited analgesia and is unlikely to provide surgical anaesthesia unless concentrations leading to anoxia are reached [4]. Nitrous oxide is an effective analgesic / anxiolytic agent that causes central nervous system depression and euphoria with little effect on the respiratory system [44]. Nitrous oxide, when administered at concentrations of $30 \%$ to $50 \%$, creates a drowsy state that appears relaxed and unrelated to the environment, but can react to stimuli. Some patients may experience amnesia, but with little change in learning or memory. At concentrations above $60 \%$, patients may experience impaired coordination, ataxia, dizziness, and increased drowsiness. Nitrous oxide concentration should not routinely exceed $50 \%$ [4]. When used at the recommended concentrations, there is a superior safety margin in which no significant morbidity and mortality is recorded [45]. As it does not irritate the respiratory tract mucosa, it does not increase the risk of bronchospasm and can be used in patients with asthma. Changes in respiratory rate and depth are not due to the direct effect of nitrous oxide, but because 
of the patient's anxiety [46]. This is particularly advantageous in the treatment of patients with cardiovascular system disorder [4].

Indications for nitrous oxide / oxygen inhalation

- The presence of dental anxiety and fear

- In cases where deep local anaesthesia such as acute pulpitis cannot be achieved

- Presence of nausea reflex, which prevents dental treatment

- Treatment of hypoplastic teeth with increased sensitivity

- Patients with long-term treatment

- As an alternative to general anaesthesia in some patients with special health care needs

- Individuals with cardiovascular disorder as they reduce anxiety and reduce myocardial oxygen demand

- Individuals with liver / kidney disease because nitrous oxide does not undergo biotransformation in the body [47].

Contraindications for nitrous oxide / oxygen inhalation

- Failure to communicate with the patient

- The presence of mask fear

- Inability to breathe through the nose

- Presence of chronic obstructive airway diseases such as emphysema, chronic bronchitis

- The presence of otitis media

- The presence of severe muscular depression, such as multiple sclerosis

- The presence of severe psychiatric illness

- The presence of behaviour / personality problems

- Being in the first trimester of pregnancy [47]. Advantages of nitrous oxide / oxygen inhalation

- It is a non-invasive method

- Can be titrated

- Absorbed

- Effect starts quickly (2-3 minutes), maximum effect reaches fast (2-5 minutes)

- Easily excreted from the body

- Fast and complete recovery in 5 minutes

- Has minimal effect on reflexes and maintains cough reflex

- It provides a partial analgesic effect [47].

Disadvantages of nitrous oxide / oxygen inhalation

- Has limited effect

- The effect is largely based on psychological relief

- Gas should be applied continuously with mask

- Nasal sealing can be prevented due to patient movement

- Nasal mask may adversely affect working in the maxillary anterior region
- Patients may reject the nasal mask

- Nitrous oxide contributes to greenhouse effect

- Potential impacts on healthcare personnel [47].

\section{Result and Conclusion}

As a result, the dentist should know which method to use and choose the appropriate sedative agent. While making this choice, the degree of difficulty of the application, cooperation of paediatric patients, anatomical and physiological characteristics of the children, age, and average processing time should be taken into consideration. In addition, the dosage, safety, duration of action, ease of administration and titration of the sedative agent to be used should be considered. Considering all these, a sedation route that provides optimum results should be preferred. It should be remembered that success in sedation can be achieved when maximum treatment is provided with the lowest dose-correct sedative agent-appropriate method.

\section{Financial disclosure}

None.

\section{Conflicts of interest}

The authors declare that they have no conflicts of interest.

\section{References}

1. Leelataweedwud P, Vann WF Jr (2001) Adverse events and outcomes of conscious sedation for pediatric patients: Study of an oral sedation regimen. J Am Dent Assoc 132: 1531-1539. [Crossref]

2. Eid H (2002) Conscious sedation in the 21st century. J Clin Pediatr Dent 26: 179-180. [Crossref]

3. Baier K, Milgrom P, Russell S, Mancl L, Yoshida T (2004) Children's fear and behavior in private pediatric dentistry practices. Pediatr Dent 26: 316-321. [Crossref]

4. Avery R, McDonald E, Dean A (2010) Mcdonald and Avery dentistry for the child and adolescent. Elsevier Health Sciences 85.

5. Kanwal F, Jamil Y, Khan H (2012) Effect of parental anxiety on child behaviour in the dental surgery. Cell 332: 922-1042.

6. Ozturk M, Ay S (2000) Conscious sedation. Cumhuriyet Dent J 3: 121-126.

7. Peretz B, Nazarian Y, Bimstein E (2004) Dental anxiety in a students' paediatric dental clinic: Children, parents and students. Int J Paediatr Dent 14: 192-198. [Crossref]

8. Onçag O, Çogulu D (2005) The Socioeconomic Status of the Family and the Effect of Education Level on Dental Anxiety in Children. J Fac Dent Ataturk Univ 32: 45-54.

9. Malamed S (2003) Sedation: A guide to patient management. (4th edn), St. Louis, Missouri, Mosby, USA.

10. Dogan C (2013) Approach to Sedation Practices in Disabled People. Turkey Clinics. $J$ Dent Clin 4: 52-58.

11. Özen B, Malamed SF, Cetiner S, Özalp N, Özer L, et al. (2012) Outcomes of moderate sedation in paediatric dental patients. Aust Dent J 57: 144-150. [Crossref]

12. Malamed S (2009) Oral sedation. Sedation: A Guide to Patient Management. ( $5^{\text {th }}$ edn), Mosby, USA, pp: 95-118.

13. Dionne RA, Yagiela JA, Coteó CJ, Donaldson M, Edwards M, et al. (2006) Balancing efficacy and safety in the use of oral sedation in dental outpatients. $J$ Am Dent Assoc 137: 502-513. [Crossref]

14. Cravero JP, Blike GT (2004) Review of Pediatric Sedation. Anesth Analg 99: 13551364. [Crossref]

15. Malamed S (2009) Intramuscular Sedation. Sedation: A Guide to Patient Management ( $5^{\text {th }}$ edn), Mosby, USA, pp:132-62.

16. Wilson KE, Thorpe RJ, McCabe JF, Girdler NM (2011) Complications Associated with Intravenous Midazolam Sedation in Anxious Dental Patients. Prim Dent Care 18: 161-66. [Crossref] 
17. Averley PA, Girdler NM, Bond S, Steen N, Steele J (2004) A randomised controlled trial of paediatric conscious sedation for dental treatment using intravenous midazolam combined with inhaled nitrous oxide or nitrous oxide/sevoflurane. Anaesthesia 59: 844852. [Crossref]

18. De Veaux CK, Montagnese TA, Heima M, Aminoshariae A, Mickel A (2016) The effect of various concentrations of nitrous oxide and oxygen on the hypersensitive gag reflex. Anesth Prog 63: 181-184. [Crossref]

19. Strunin L (2007) Intravenous conscious sedation for dental treatment: Am I My Brother's Keeper? Anaesthesia 62: 645-647. [Crossref]

20. Guideline S (2008) Scottish Intercollegiate Guidelines Network. SIGN Guideline 58: Safe Sedation of Children Undergoing Diagnostic and Therapeutic Procedures. Paediatr Anaesth 18: 11-12. [Crossref]

21. Malamed S (2009) Sublingual, transdermal, and intranasal sedation. sedation: A Guide to Patient Management ( $5^{\text {th }}$ edn), Mosby, USA, pp:124-3.

22. Wolfe TR, Braude DA (2010) Intranasal medication delivery for children: A brief review and update. Pediatrics 126: 532-537. [Crossref]

23. Shanmugaavel AK, Asokan S, Baby JJ, Priya G, Gnana Devi J (2016) Comparison of behavior and dental anxiety during intranasal and sublingual midazolam sedation-A randomized controlled trial. J Clin Pediatr Dent 40: 81-87. [Crossref]

24. Berthold C (2007) Enteral sedation: safety, efficacy, and controversy. Compend Contin Educ Dent 28: 264-271. [Crossref]

25. Faytrouny M, Okte Z, Kucukyavuz Z (2007) Comparison of two different dosages of hydroxyzine for sedation in the paediatric dental patient. Int J Paediatr Dent 17: 378382. [Crossref]

26. Wilson S, Easton J, Lamb K, Orchardson R, Casamassimo P (2000) A retrospective study of chloral hydrate, meperidine, hydroxyzine, and midazolan regimens used to sedate children for dental care. Pediatr Dent 22: 107-112. [Crossref]

27. Mittal N, Gauba K, Goyal A, Kapur A (2014) Pediatric dental sedation practice: Evolution and current state-of-the-art. J Postgrad Med Educ Res 48: 139.

28. Eberson CP, Hsu RY, Borenstein TR (2015) Procedural sedation in the emergency department. J Am Acad Orthop Surg 23: 233-242. [Crossref]

29. Papineni A, Lourenço-Matharu L, Ashley PF (2014) Safety of oral midazolam sedation use in paediatric dentistry: a review. Int J Paediatr Dent 24: 2-13. [Crossref]

30. Papineni McIntosh A, Ashley PF, Lourenco-Matharu L (2015) Reported side effects of intravenous midazolam sedation when used in paediatric dentistry: A review. Int $J$ Paediatr Dent 25: 153-164. [Crossref]

31. Isık B, Baygin Ö, Bodur H (2008) Effect of drinks that are added as flavoring in oral midazolam premedication on sedation success. Paediatr Anaesth 18: 494-500. [Crossref]
32. Singh N, Pandey RK, Saksena AK, Jaiswal JN (2003) A comparative evaluation of oral midazolam with other sedatives as premedication in pediatric dentistry. J Clin Pediatr Dent 26: 161-64. [Crossref]

33. Malamed S (2009) Pharmacology. Sedation: A guide to patient management ( $5^{\text {th }}$ edn) Mosby, USA, pp: 316-54.

34. Giovannitti Jr, Joseph A (1995) Dental anesthesia and pediatric dentistry. Anesth Prog 42: 95-99.

35. Malamed S (2009) The pediatric patient. Sedation: A Guide to Patient Management (5 edn), Mosby, USA, pp: 495-513.

36. Coté J, Karl W, Notterman A, Weinberg A, McCloskey C (2000) Adverse Sedation Events in Pediatrics: Analysis of Medications Used for Sedation. Pediatrics 106: 633644. [Crossref]

37. Craven R (2007) Ketamine. Anaesthesia 62: 48-53. [Crossref]

38. Wathen JE, Roback MG, Mackenzie T, Bothner JP (2000) Does midazolam alte the clinical effects of intravenous ketamine sedation in children? A double-blind, randomized, controlled, emergency department trial. Ann Emerg Med 36: 579-588. [Crossref]

39. Hosey MT, (2002) UK National Clinical Guidelines in Pediatric Dentistry UK National Clinical Guidelines in Paediatric Dentistry. Managing anxious children: the use of conscious sedation in paediatric dentistry. Int J Paediatr Dent 12: 359-372. [Crossref]

40. Hosey M, Makin A, Jones R, Gilchrist F, Carruthers M (2004) Propofol intravenous conscious sedation for anxious children in a specialist paediatric dentistry unit. Int $J$ Paediatr Dent 14: 2-8. [Crossref]

41. Baygın Ö, Işık B (2010) Nitrous Oxide / Oxygen Sedation in Pediatric Dentistry. Clin Dent Res 34: 53-61.

42. Hulland SA, Freilich MM, Sàndor GK (2002) Nitrous oxide-oxygen or oral midazolam for pediatric outpatient sedation. Oral Surg Oral Med Oral Pathol Oral Radiol Endod 93: 643-46. [Crossref]

43. Isık B, Baygın O, Bodur H (2008) Oral Midazolam Premedication in Children with High Anxiety Level: Effectiveness of Different Doses. J Anesth 16: 34-40.

44. AAPD (2013) Guideline on use of nitrous oxide for pediatric dental patients. Pediatr Dent 35: 174-178. [Crossref]

45. Holroyd I (2008) Conscious sedation in pediatric dentistry. A short review of the current uk guidelines and the technique of inhalational sedation with nitrous oxide. Paediatr Anaesth 18: 13-17. [Crossref]

46. Malamed S (2009) Pharmacology, anatomy, and physiology, sedation: A guide to patient management ( $5^{\text {th }}$ edn $)$, Mosby, USA, pp:190-201.

47. AAPD (2013) Guideline on use of nitrous oxide for pediatric dental patients. Pediatr Dent 35: 174-178. [Crossref]

Copyright: (C2020 Oznurhan F. This is an open-access article distributed under the terms of the Creative Commons Attribution License, which permits unrestricted use, distribution, and reproduction in any medium, provided the original author and source are credited. 